\title{
Safety and Efficacy of Vitamin K Antagonists vs. Novel Oral Anticoagulants in Patients With Left Ventricular Thrombus: A Meta-Analysis
}

\author{
He Xuan ${ }^{1+}$, Yi-Ming Chen ${ }^{1+}$, Yun-Lang Dai ${ }^{2+}$, Jing Zhou ${ }^{2}$, Yu-Feng Jiang ${ }^{1}$ and \\ Ya-Feng Zhou ${ }^{1 *}$
}

${ }^{1}$ Department of Cardiology, Dushu Lake Hospital Affiliated to Soochow University, Suzhou, China, ${ }^{2}$ Department of Cardiology, The First Affiliated Hospital of Soochow University, Suzhou, China

OPEN ACCESS

Edited by:

Tetsuro Miyazaki,

Juntendo University Urayasu

Hospital, Japan

Reviewed by:

Philipp Diehl,

University Heart Center

Freiburg, Germany

Florian Schlotter,

Herzzentrum Leipzig, Helios

Kliniken, Germany

${ }^{*}$ Correspondence:

Ya-Feng Zhou

zhouyafeng73@126.com

†These authors have contributed equally to this work

Specialty section:

This article was submitted to

Atherosclerosis and Vascular

Medicine,

a section of the journal

Frontiers in Cardiovascular Medicine

Received: 06 December 2020 Accepted: 30 March 2021

Published: 29 April 2021

Citation:

Xuan H, Chen Y-M, Dai Y-L, Zhou J, Jiang Y-F and Zhou Y-F (2021) Safety and Efficacy of Vitamin $K$ Antagonists

vs. Novel Oral Anticoagulants in

Patients With Left Ventricular

Thrombus: A Meta-Analysis.

Front. Cardiovasc. Med. 8:636491.

doi: 10.3389/fcvm.2021.636491
Aims: A meta-analysis was conducted to evaluate the safety and efficacy of novel oral anticoagulants (NOACs) compared with vitamin $\mathrm{K}$ antagonists (VKAs) in patients with left ventricular thrombus (LVT).

Methods and Results: We searched PubMed, Web of Science, and Cochrane Library for cohort studies comparing the use of VKAs vs. NOACs for the treatment of LVT from the earliest date available to September 30, 2020. The predetermined efficacy and safety outcomes included thromboembolic events, resolution of LVT, clinically significant bleedings, and all-cause death. Fixed-effects model was used to estimate the pooled effects. Publication bias analyses and sensitivity analyses were conducted to check the robustness of results. A total of 6 studies enrolling 837 patients (mean age 60.2 \pm 1.6 years; $77.2 \%$ were male) were included. We found no significant differences in thromboembolic events [relative risk (RR) 1.69, 95\% confidence interval (CI) 0.94-3.06, $P 0.08, I^{2} 12.7 \%$ ], the rate of resolution of thrombus (RR $1.08,95 \% \mathrm{Cl} 0.96-1.21, P$ $0.21, \mathrm{I}^{2} 4.8 \%$ ), and clinically significant bleedings (RR 0.70, 95\% Cl 0.37-1.32, P 0.27 , $\left.\mathrm{I}^{2} 0 \%\right)$ between the VKAs and NOACs group. Additionally, no significant difference in all-cause mortality was found between the two groups (RR 1.24, 95\% Cl 0.79-1.96, $P$ $\left.0.35, I^{2} 0.0 \%\right)$. Sensitivity analyses, using the " 1 -study removed" method, detected no significant differences.

Conclusion: NOACs and VKAs have similar efficacy and safety in treating LVT, prompting the inference that NOACs are the possible alternatives of VKAs in LVT therapy.

Keywords: novel oral anticoagulant, vitamin $\mathrm{K}$ antagonist, bleeding, thromboembolic events, left ventricular thrombus

\section{INTRODUCTION}

Left ventricular thrombus (LVT) is a common complication of acute myocardial infarction (MI) $(1,2)$, and is also observed in patients with non-ischemic cardiomyopathies with severe left ventricular systolic dysfunction (3). Previous studies have suggested that LVT can significantly increase the risk of developing embolic events by 5.5 -fold $(4,5)$. Current guidelines recommend the use of vitamin K antagonists (VKAs) in patients with post-MI LVT $(6,7)$. However, as its slow 
onset and the fluctuation of drug concentration, regular monitoring of international normalized ratio (INR) and constant adjustment for warfarin dosage are required to achieve a safe and efficacious outcome, which may potentially lead to decreased patient compliance (8).

Novel oral anticoagulants (NOACs) are the first-line treatments against thromboembolic events in patients with non-valvular atrial fibrillation (9). Considering the efficacy and safety of NOACs, NOACs are sometimes used off-label for anticoagulation therapy in patients with LVT in clinical practice. To date, most studies of NOACs for the management of thrombotic events in patients with LVT are case reports and cohort studies with limited sample sizes.

So far, several studies have been conducted to compare the safety and efficacy of VKAs vs. NOACs for anticoagulation therapy in patients with LVT. Recently, a meta-analysis performed by Cochran et al. showed non-inferiority of NOACs in treating LVT compared to VKAs (10); however, half of the studies included were abstracts without full manuscript, which may hamper the generalization of the result. Moreover, several studies have been published after the time of Cochran's metaanalysis (11-13). Hence, we conducted the meta-analysis, which included only studies published in peer-reviewed journals, to evaluate the safety and efficacy of NOACs compared with VKAs in patients with LVT.

\section{METHODS}

\section{Search Strategy and Selection Criteria}

We systemically searched PubMed, Web of Science, and Cochrane Library database for relevant studies published before September 30, 2020. Search items included "Factor Xa inhibitor", "direct oral anticoagulant", "NOAC", "DOAC", "dabigatran", "rivaroxaban", "edoxaban", "apixaban” combined with "vitamin $\mathrm{K}$ antagonist", "Warfarin" and "left ventricular thrombi*". We also checked the reference lists of obtained articles to avoid omissions. Abstracts, meeting proceedings, and private communications were not included in this study.

The articles included should meet the following inclusion criteria: (1) published studies in English in peer-reviewed journals; (2) adult patients diagnosed with LVT; (3) data about the efficacy and safety in LVT patients taking VKAs or NOACs was available. For studies with overlapping cohorts, the article with the most comprehensive data would be included for analysis.

We followed the Preferred Reporting Items for Systematic Reviews and Meta-Analysis (PRISMA) guidelines (14) and the Cochrane Handbook for Systematic Reviews to perform and report this systematic review and meta-analysis.

\section{Data Extraction and Quality Assessment}

YC and HX independently screened all retrieved articles and discussed it with a third investigator (YD) when facing disagreements. Inclusion and exclusion criteria, baseline characteristics of the included patients, treatment methods, and all outcomes in each group were extracted. We extracted the most comprehensively adjusted or unadjusted hazard ratios
(HRs) and 95\% confidence intervals (CIs) from Cox proportional hazards analysis. Otherwise, we extracted an exact number of specific outcomes.

We used the Newcastle-Ottawa Quality Assessment Scale for Cohort Studies (NOS) (15) to assess the bias of each study. According to NOS, each study would get $0 \sim 4$ points in selecting of the study groups, $0 \sim 2$ points in the comparability of the study group, and $0 \sim 3$ points in the ascertainment of the outcome of interest. Then studies were classified into high (total $0 \sim 4$ points), medium (total $5 \sim 7$ points), and low (total $8 \sim 9$ points) risk of bias.

\section{Outcomes Assessment}

The efficacy endpoints were thromboembolic events, consisting of stroke, transient ischemic attack, and peripheral artery embolism during the period of observation, and resolution of LVT (defined as no evidence of thrombus on repeat imaging). Safety endpoints were clinically significant bleedings (defined as in the individual article) and all-cause mortality.

\section{Data Analysis}

We used relative risks (RRs) with the 95\% CIs to compare the differences between VKAs and NOACs group for the metaanalyses. Q test was used to evaluate the heterogeneity of included studies with $\mathrm{I}^{2}$ and $P$-values. The pooled RRs were calculated by the fixed-effects model according to the Mantel-Haenszel method or by the random-effects model with Der Simonian and Laird method for studies with present heterogeneity $\left(\mathrm{I}^{2}>50 \%\right.$ or $P$ value $<0.05)$. We detected the publication bias of articles by drawing the funnel plot with the Egger test. Furthermore, the contour-funnel plot in conjunction with the trim-and-fill method was used to identify the causes of asymmetry observed in a funnel plot (16). In addition, we conducted sensitivity analyses with the "1-study removed" method to check the credibility of the results. All data analysis was carried out using Stata/SE 15.1.

\section{RESULTS}

\section{Results of the Literature Search}

Three hundred and one studies were enrolled after searching three databases mentioned above. After the removal of duplicates, there were remaining 182 articles. Eighty-two articles were excluded because they were reviews, case reports, or letters. In the left 100 articles, 57 studies were excluded for not investigating patients with LVT, 30 studies for without comparison between VKAs and NOACs, and 1 for animal study. Finally, we performed full-text screening on the remaining 12 documents: 3 of them were excluded for not being done (17-19), and 3 were duplicate researches (20-22). Then six studies left for subsequent data analyses with sample sizes ranged from 59 to 421 $(10-13,23,24)$. The complete screening process was shown as a flow chart in Figure 1.

\section{Study Characteristics and Quality}

The patient characteristics were shown in Table 1. A total of 837 patients in 6 studies were included in this meta-analysis. Overall, $77.2 \%$ were male, and the mean age of patients was 


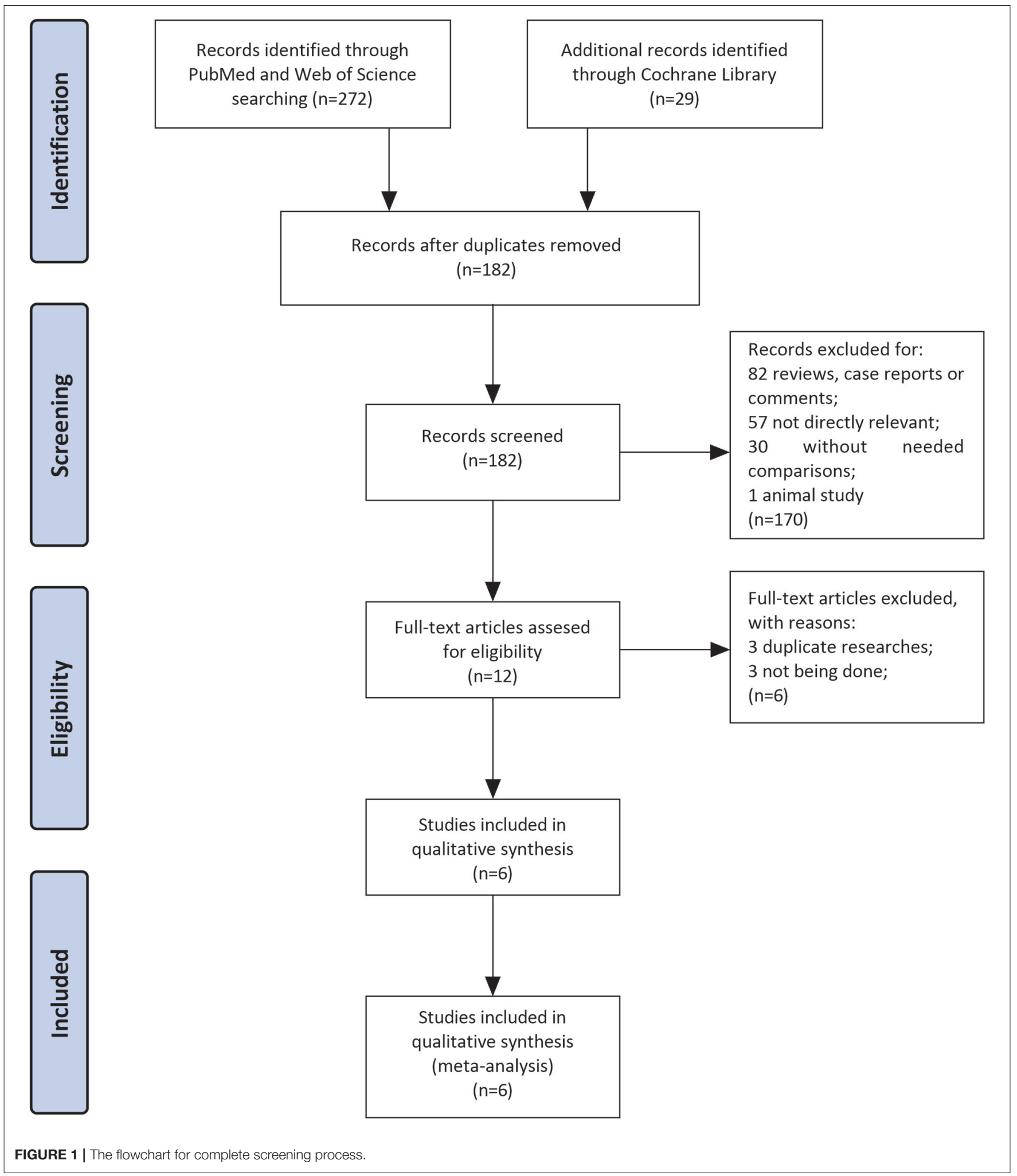

$60.2 \pm 1.6$ years old. Transthoracic echocardiography (TTE) was the primary method for the diagnosis of LVT, and cardiac magnetic resonance imaging (CMR) was also performed in some patients to make the diagnosis. Ischemic cardiomyopathy (67.9\%) dominated in the etiology of LVT. Of the $234(28.0 \%)$ patients who received NOACs, rivaroxaban $(43 / 99,43.4 \%)$ and 
apixaban (50/99, 50.5\%) were preferred NOACs; however, details of anticoagulant regimens were not reported in the remaining 135 subjects $(10,24)$. The doses of NOACs chosen in the included studies were identical to those applied in the primary stroke prevention for patients with atrial fibrillation. All included studies were of low to medium bias, and details of NOS assessment can be found in Table $\mathbf{1}$.

\section{Efficacy Endpoints}

The resolution of LVT and thromboembolic events were reported in all six studies. During the follow-up, thromboembolic events and resolution of LVT occurred in 54 (6.5\%) and 483 (58.3\%) subjects, respectively. No difference was detected between the NOACs group and the VKAs group (RR 1.08, 95\% CI 0.96-1.21, $P 0.21, \mathrm{I}^{2} 4.8 \%$ ) for the resolution of LVT. As for thromboembolic events, there was no significant difference between the two groups (RR 1.69, 95\% CI 0.94-3.06, P $0.08, \mathrm{I}^{2} 12.7 \%$ ), and this conclusion did not change after excluding 64 individuals who switched treatment (RR 1.35, 95\% CI 0.72-2.51, P 0.35, $\mathrm{I}^{2}$ $0.0 \%$ ) (Supplementary Figure 1). Forest plots for comparisons of resolution of LVT and thromboembolic events between two groups were shown in Figure 2.

\section{Safety Outcomes}

In LVT therapy, clinically significant bleedings were considered as the essential parameters for evaluating safety. Five studies provided the number of bleeding events in both groups (1013,24 ). The occurrence of clinically significant bleedings (as defined in the individual study in Supplementary Table 1) was not significantly different between VKAs and NOACs group (RR $0.70,95 \%$ CI $0.37-1.32, P 0.27, \mathrm{I}^{2} 0.0 \%$ ) (Figure 2C). As for allcause death, Iqbal et al. (12), Cochran et al. (10) and Robinson et al. (24) provided the relevant data, and no significant difference can be detected (RR 1.24, 95\% CI 0.79-1.96, P $0.35, \mathrm{I}^{2} 0.0 \%$ ) (Figure 2D).

\section{Publication Bias}

Funnel plots for individual endpoint were drawn, and no asymmetry was found when evaluated with the Egger's test except for thromboembolic events ( $\mathrm{P}$ for thromboembolic events = $0.01, \mathrm{P}$ for bleeding $=0.52, \mathrm{P}$ for resolution of thrombosis $=$ 0.15 , P for death $=0.12$ ) (Figure 3 ). However, the contourfunnel plot in conjunction with the trim-and-fill method implied the observed asymmetry in the funnel plot for thromboembolic events might not be due to publication bias, as no "missing" studies were indicated in the Supplementary Figure 2.

\section{Sensitivity Analyses}

Sensitivity analyses by sequentially removing a single study were performed, detecting no significant difference except for pooling the data of thromboembolic events after removing the study by Robinson et al. (24) (Figure 4).

\section{DISCUSSION}

LVT is a severe complication of cardiovascular diseases, most commonly occurred in patients with anterior myocardial

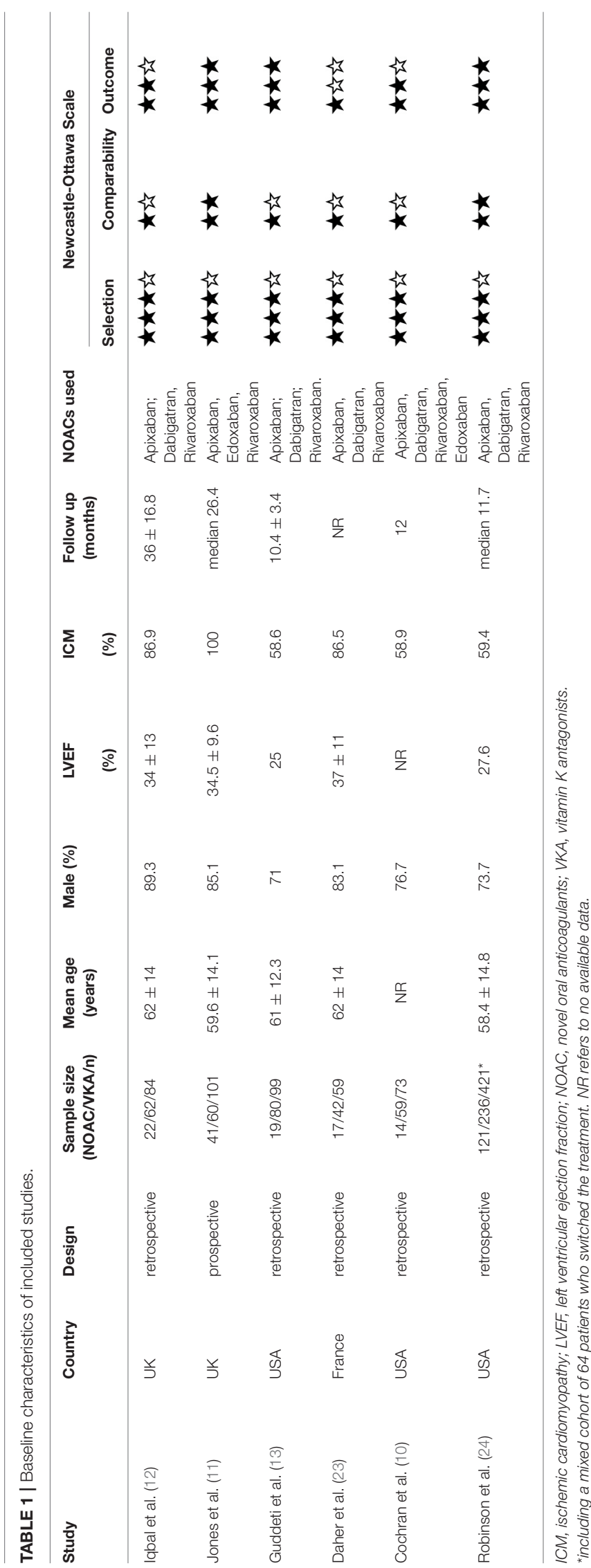




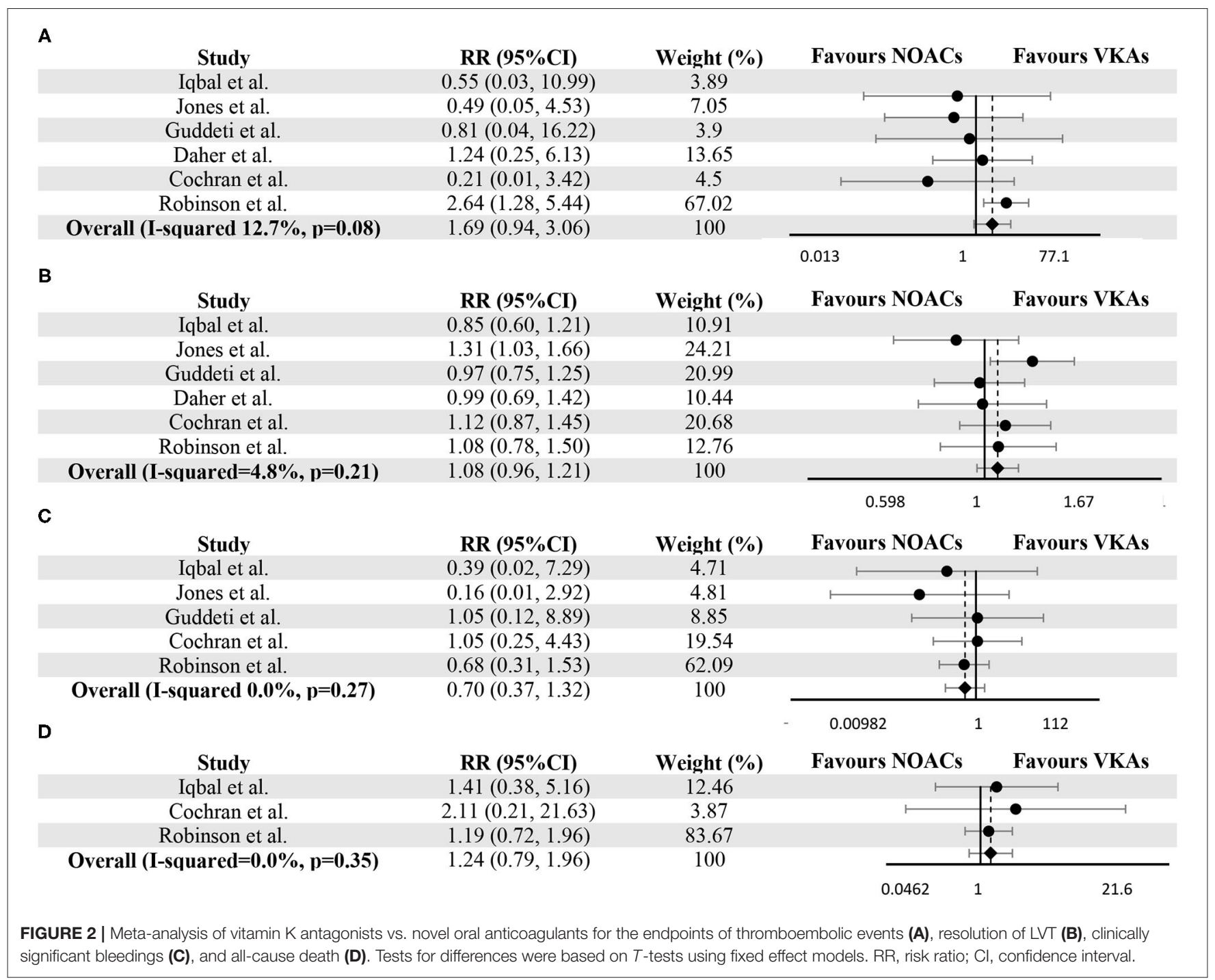

infarction. The overall incidence of developing LVT in patients with acute $\mathrm{MI}$ is about $17 \%$ in the thrombolytic era and has decreased to $3 \%$ with the universal access to percutaneous coronary intervention (PCI) (25). Nevertheless, the incidence of LVT in patients with anterior MI is still high (9\%) (26). Besides, LVT is closely related to several adverse cardiovascular events during the 1-year follow-up (27).

According to the American College of Cardiology Foundation/American Heart Association Guidelines, patients with ST-segment elevation myocardial infarction (STEMI) and LVT are recommended to receive anticoagulant therapy with VKAs for 3 months, similar to suggestions in the AHA/American Stroke Association 2014 guidelines on stroke prevention $(6,7)$. While the European Society of Cardiology 2017 STEMI guidelines suggest the use of anticoagulants without specific recommendations on strategies to prevent LVT (28). Periodic monitoring of TTE is recommended in all three guidelines.

VKAs block the enzyme vitamin K epoxide reductase, thereby inhibiting reactivation of vitamin $\mathrm{K} 1$, which is essential to synthesize coagulation factors II, VII, IX, and X. Besides, VKAs can also inhibit anticoagulant proteins $\mathrm{C}$ and protein $\mathrm{S}$ but to a lesser extent. Warfarin is the most commonly used VKA. However, apart from its slow onset and the fluctuation of drug concentration, warfarin is known to interact with many common drugs and certain foods (29). Thus, there are well-recognized difficulties for the management of anticoagulation with VKAs.

NOACs are highly selective $\mathrm{Xa}$ factor or direct thrombin inhibitors. Due to the specificity of reversely combining with the $\mathrm{Xa}$ factor, NOACs can be administrated without the need for routine coagulation monitoring, which could potentially increase patient compliance (30). Although NOACs are less effective in the prevention of systematic thromboembolic events in patients with mechanic valves (31), the long-term use of NOACs as the first-line anticoagulants in patients with left atrial thrombus and non-valvular atrial fibrillation has been demonstrated to have satisfying efficacy and safety (32). However, due to the intrinsic mechanistic differences between LVT and left atrial thrombus [the former may involve both the blood stasis and endocardial 


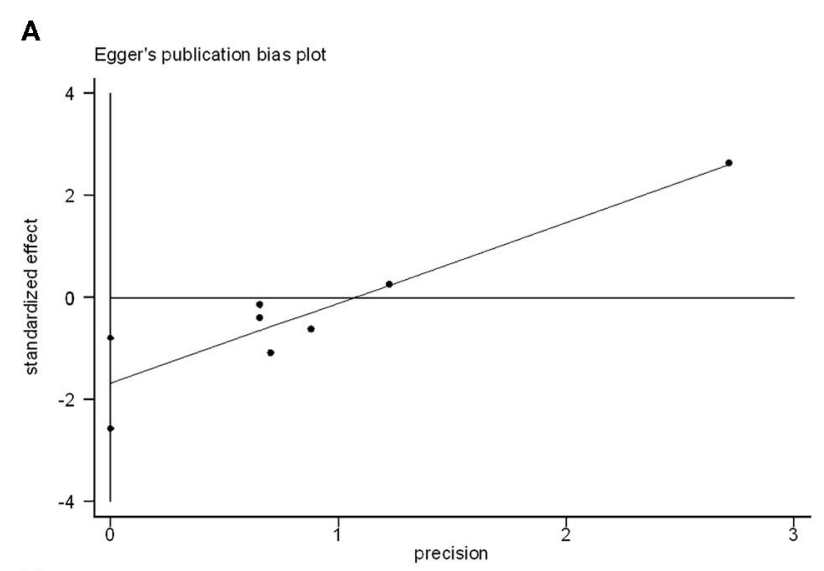

。

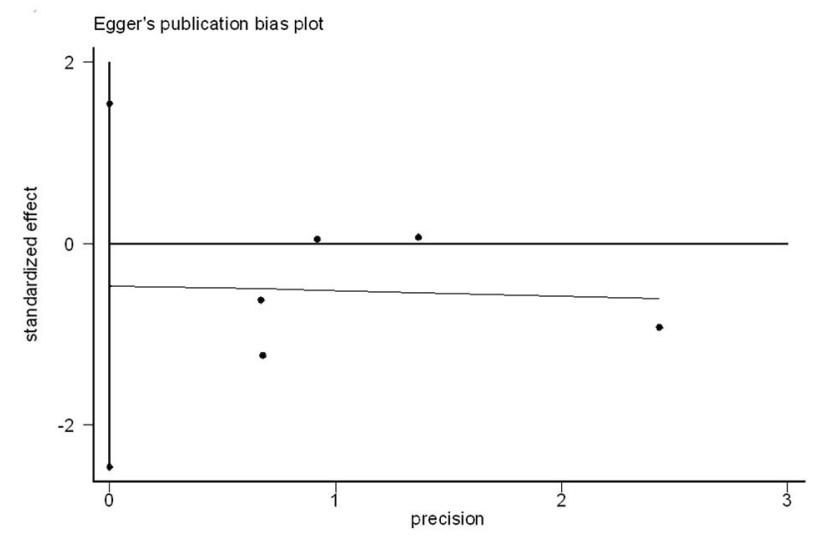

B

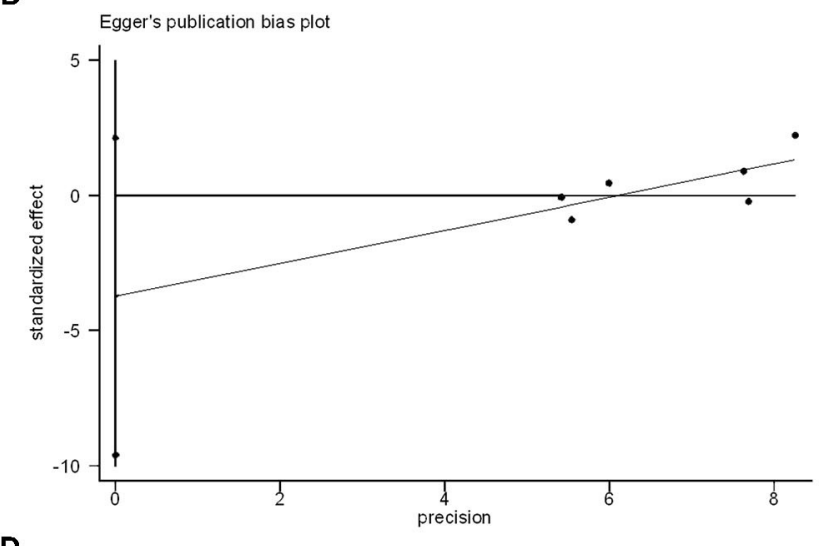

D

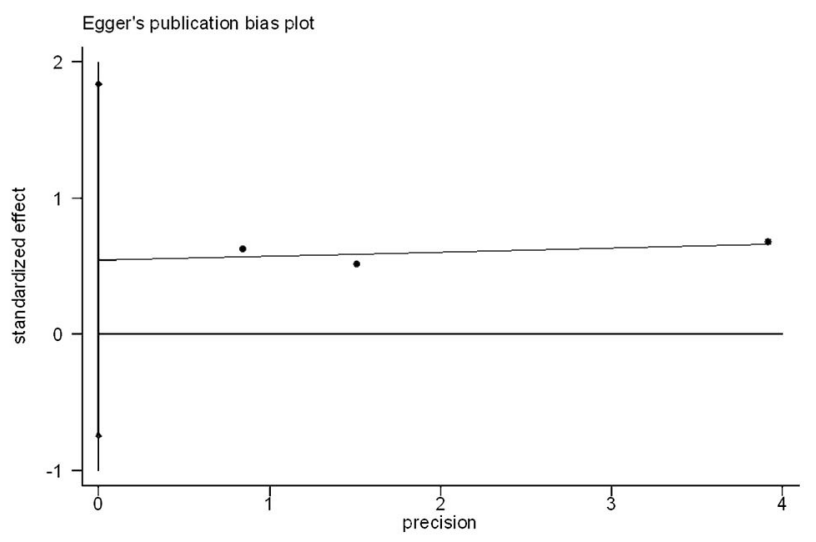

FIGURE 3 | Publication bias analysis of vitamin K antagonists vs. novel oral anticoagulants for the endpoints of thromboembolic events (A), resolution of LVT (B), clinically significant bleedings (C), and all-cause death (D) by Egger's test.

damage (33)], clinical evidence of anticoagulant use derived from studies based on patients with atrial fibrillation may not be applicable to patients with LVT (31).

Recently, some studies have noted that NOACs may have similar efficacy to warfarin in the treatment of LVT but reached opposite conclusions. The study by Robinson et al. showed the superiority of VKAs over NOACs for the thromboembolic events; nevertheless, the superiority disappeared when the analysis was restricted to patients who did not switch treatment (RR 1.99, 95\% CI $0.91-4.35, P 0.08)$ or based on intention-totreatment analysis (RR 1.42, 95\% CI 0.68-1.92, P 0.35) (24). In this meta-analysis, after pooling 837 patients of LVT treated with VKAs or NOACs from six studies, no difference was found in the resolution of LVT (RR 1.08, 95\% CI 0.96-1.21, P 0.212, $\mathrm{I}^{2}$ $4.8 \%$ ) and thromboembolic events (RR 1.69, 95\% CI 0.94-3.06, $\left.P 0.08, \mathrm{I}^{2} 12.7 \%\right)$ between VKAs and NOACs group, indicating the similar efficacy of VKAs and NOACs in LVT therapy. As for analyses of clinically significant bleedings and death, no significant differences were detected. The heterogeneity tests for the results were low, suggesting a high level of clinical evidence. These results support the use of NOACs have similar safety and efficacy profile in the treatment of LVT.
Doubts on the efficacy of NOACs for the treatment of LVT exist, as there may be intrinsic mechanistic differences between LVT and thrombus associated with atrial fibrillation (33). However, thrombosis associated with endocardial changes in myocardial infarction should theoretically be transient and is different from that related to mechanical valves, in which case NOACs should be avoided (31). Thus, although current evidence suggests NOACs achieve similar clinical outcomes compared with VKAs for the treatment of LVT, further large-scale clinical trials are needed to establish more robust clinical evidence.

Concomitant antiplatelet therapy was broadly used in the included studies (65\% 92.1\%); furthermore, 38\% 69.3\% of patients were prescribed with dual antiplatelet therapy for other indications (e.g., acute coronary syndrome, percutaneous coronary intervention) $(11-13,24)$. Co-prescribed antithrombotic therapy, notably triple antithrombotic strategy, undoubtedly increases the bleeding risk $(11,34)$; however, this can be alleviated by the novel antithrombotic strategy indicated in several recent researches, which revealed the superiority of the regimen including a NOAC and a $\mathrm{P}_{2} \mathrm{Y}_{12}$ inhibitor over traditional triple antithrombotic strategy $(35,36)$. 
A

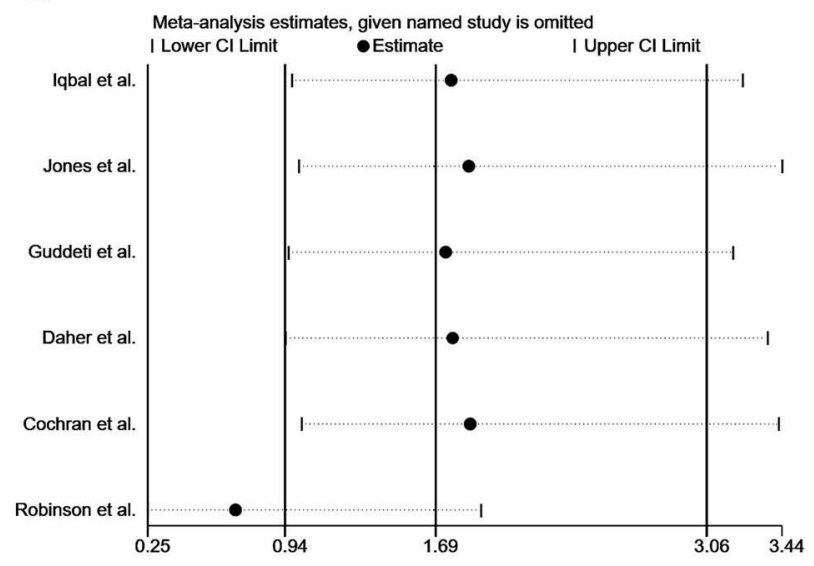

C

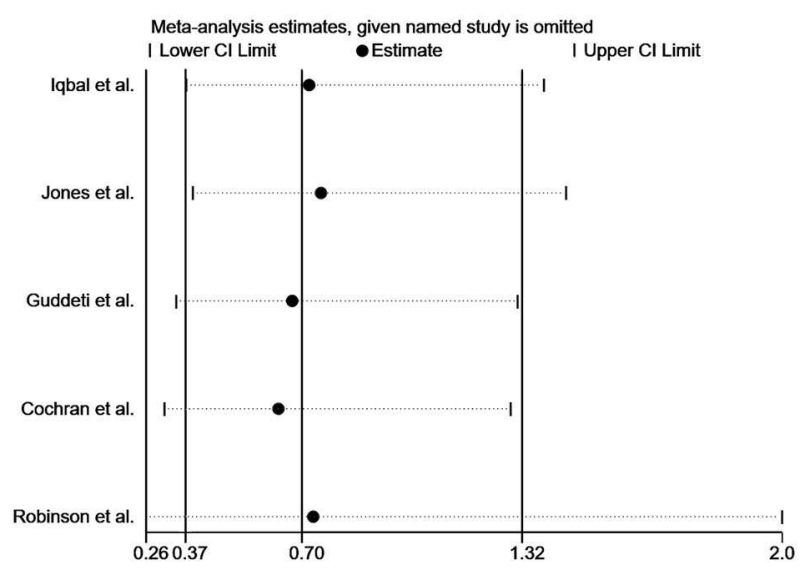

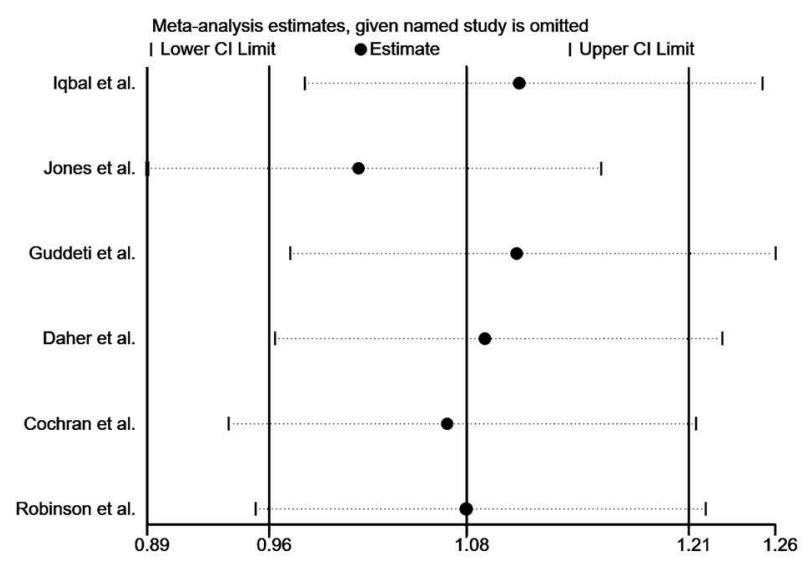

D

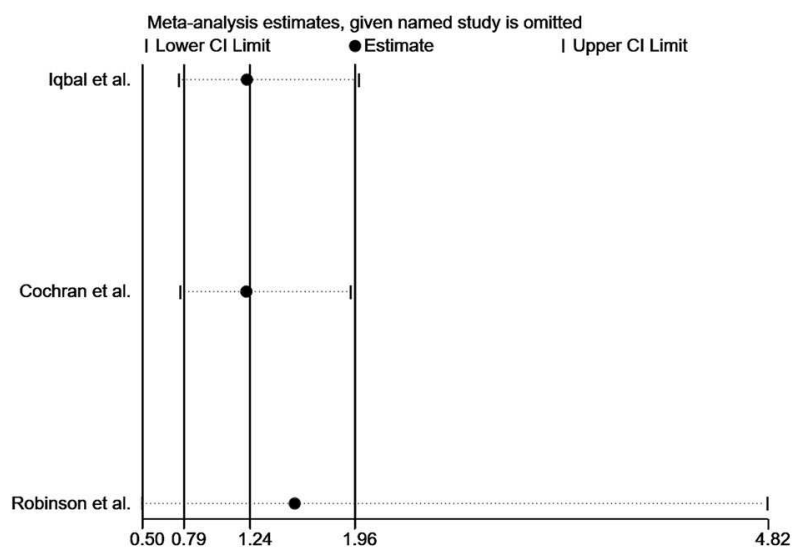

FIGURE 4 | Sensitivity analysis of vitamin K antagonists vs. novel oral anticoagulants for the endpoints of thromboembolic events (A), resolution of LVT (B), clinically significant bleedings (C), and all-cause death (D) with the "1-study removed" method.

Today, we are facing two main dilemmas in the anticoagulation of ventricular thrombus. One is the choice of anticoagulation intensity. The therapeutic dose of NOACs for venous thromboembolism is higher than the prophylactic dose for stroke prevention in patients with non-valvular atrial fibrillation, making it difficult to extrapolate the appropriate therapeutic dose for LVT. Moreover, studies have shown that the use of warfarin to control the INR to 3-4 ameliorates the resolution of ventricular thrombus in patients who failed in NOACs therapy (23). Another dilemma is the duration of anticoagulation. It may be challenging to make the decision of anticoagulation discontinuation in cardiomyopathies (e.g., dilated cardiomyopathy) that cannot be fully recovered and often have no acute event time point.

\section{Limitations}

Our meta-analysis has several limitations. Firstly, studies pooled in our research were retrospective studies with small sample sizes; further prospective, large-scale, randomized clinical trials are needed to establish more robust clinical evidence. Secondly, all six included studies used TTE rather than CMR as a primary diagnostic standard, which is likely to introduce misdiagnoses. Thirdly, although antiplatelet use was a significant confounder for the outcomes, we cannot fully adjust our results due to the lack of individual data.

\section{CONCLUSION}

In this meta-analysis to investigate the differences between NOACs and VKAs for the treatment of LVT, no differences were found in the efficacy and safety, which inferred that NOACs might be a promising candidate for LVT therapy.

\section{DATA AVAILABILITY STATEMENT}

The original contributions presented in the study are included in the article/Supplementary Material, further inquiries can be directed to the corresponding authors. 


\section{AUTHOR CONTRIBUTIONS}

HX: formal analysis, visualization, and writing-review \& editing. Y-MC: investigation, data curation, and writing original draft. Y-LD: conceptualization, project administration, and methodology. JZ: validation and resources. Y-FJ: software. Y-FZ: supervision and funding acquisition. All authors contributed to the article and approved the submitted version.

\section{FUNDING}

This work was supported by grants from National Natural Science Foundation of China (81873486), Natural Scientific Fund of Jiangsu province (BK20161226), Jiangsu Province's Key Provincial Talents Program (ZDRCA2016043), Jiangsu Province's 333 High-Level Talents Project (BRA2017539). The funders had

\section{REFERENCES}

1. Weinsaft JW, Kim J, Medicherla CB, Ma CL, Codella NC, Kukar N, et al. Echocardiographic algorithm for post-myocardial infarction LV thrombus: a gatekeeper for thrombus evaluation by delayed enhancement CMR. JACC Cardiovasc Imag. (2016) 9:505-15. doi: 10.1016/j.jcmg.2015.06.017

2. McCarthy CP, Vaduganathan M, McCarthy KJ, Januzzi Jr JL, Bhatt DL, McEvoy JW. Left ventricular thrombus after acute myocardial infarction: screening, prevention, and treatment. JAMA Cardiol. (2018) 3:6429. doi: 10.1001/jamacardio.2018.1086

3. Habash F, Vallurupalli S. Challenges in management of left ventricular thrombus. Ther Adv Cardiovasc Dis. (2017) 11:20313. doi: $10.1177 / 1753944717711139$

4. Stratton JR, Resnick AD. Increased embolic risk in patients with left ventricular thrombi. Circulation. (1987) 75:100411. doi: 10.1161/01.CIR.75.5.1004

5. Vaitkus PT, Barnathan ES. Embolic potential, prevention and management of mural thrombus complicating anterior myocardial infarction: a metaanalysis. J Am Coll Cardiol. (1993) 22:1004-9. doi: 10.1016/0735-1097(93)9 0409-T

6. O'Gara PT, Kushner FG, Ascheim DD, Casey DE, Chung MK, de Lemos JA, et al. 2013 ACCF/AHA guideline for the management of st-elevation myocardial infarction: a report of the American college of cardiology foundation/American heart association task force on practice guidelines. J Am Coll Cardiol. (2013) 61:e78-140. doi: 10.1016/j.jacc.2012.11.019

7. Kernan WN, Ovbiagele B, Black HR, Bravata DM, Chimowitz MI, Ezekowitz $\mathrm{MD}$, et al. Guidelines for the prevention of stroke in patients with stroke and transient ischemic attack: a guideline for healthcare professionals from the American heart association/American stroke association. Stroke. (2014) 45:2160-236. doi: 10.1161/STR.0000000000000024

8. Wells PS, Holbrook AM, Crowther NR, Hirsh J. Interactions of warfarin with drugs and food. Ann Intern Med. (1994) 121:676-83. doi: 10.7326/0003-4819-121-9-199411010-00009

9. Hindricks G, Potpara T, Dagres N, Arbelo E, Bax JJ, Blomström-Lundqvist C, et al. 2020 ESC Guidelines for the diagnosis and management of atrial fibrillation developed in collaboration with the European association of cardio-thoracic surgery (EACTS). Eur Heart J. (2020) 42:373-498. doi: 10.1093/eurheartj/ehaa612

10. Cochran JM, Jia X, Kaczmarek J, Staggers KA, Rifai MA, Hamzeh IR, et al. Direct oral anticoagulants in the treatment of left ventricular thrombus: a retrospective, multicenter study and meta-analysis of existing data. J Cardiov Pharmacol Therap. (2020) 26:173-8. doi: 10.1177/1074248420967644

11. Jones DA, Wright P, Alizadeh MA, Fhadil S, Rathod KS, Guttmann O, et al. The use of novel oral anti-coagulant's (NOAC) compared to vitamin $\mathrm{K}$ Antagonists (Warfarin) in patients with left ventricular thrombus after no roles in study design, data collection and analysis, decision to publish, or preparation of the manuscript.

\section{SUPPLEMENTARY MATERIAL}

The Supplementary Material for this article can be found online at: https://www.frontiersin.org/articles/10.3389/fcvm. 2021.636491/full\#supplementary-material

Supplementary Figure 1 | Meta-analysis of vitamin K antagonists vs. novel oral anticoagulants for the endpoints of thromboembolic events after excluding 64 individuals who switched treatment. Tests for differences were based on T tests using fixed effect models.

Supplementary Figure 2 | Contour-funnel plot in conjunction with the trim-and-fill method used to identify the causes of the observed asymmetry in the funnel plot for thromboembolic events.

Supplementary Table 1 | Definitions for clinically significant bleedings in included studies.

acute myocardial infarction (AMI). Euro Heart $J$ Cardiov Pharmacother. (2020). doi: 10.1093/ehjcvp/pvaa096. [Epub ahead of print].

12. Iqbal H, Straw S, Craven TP, Stirling K, Wheatcroft SB, Witte KK. Direct oral anticoagulants compared to vitamin $\mathrm{K}$ antagonist for the management of left ventricular thrombus. ESC Heart Failure. (2020). doi: 10.1002/ehf2.12718

13. Guddeti RR, Anwar M, Walters RW, Apala D, Pajjuru V, Kousa $\mathrm{O}$, et al. Treatment of left ventricular thrombus with direct oral anticoagulants: a retrospective observational study. Am J Med. (2020) 133:1488-91. doi: 10.1016/j.amjmed.2020.05.025

14. Moher D, Liberati A, Tetzlaff J, Altman DG. Preferred reporting items for systematic reviews and meta-analyses: the PRISMA statement. Bmj. (2009) 339:b2535. doi: 10.1136/bmj.b2535

15. Wells GSB, O'Connell D, Peterson J, Welch V, Losos M, Tugwell P. The Newcastle-Ottawa Scale (NOS) for Assessing the Quality of Nonrandomized Studies in Meta-Analyses. (2017). Available online at: http://wwwohrica/ programs/clinical_epidemiology/oxfordasp 2013

16. Peters JL, Sutton AJ, Jones DR, Abrams KR, Rushton L. Contourenhanced meta-analysis funnel plots help distinguish publication bias from other causes of asymmetry. J Clin Epidemiol. (2008) 61:9916. doi: 10.1016/j.jclinepi.2007.11.010

17. Bass M, Page RL, Kiser TH, McIlvennan CK, Allen LA, Wright G, et al. Comparative effectiveness of direct oral anticoagulants and warfarin for the treatment of left ventricular thrombus. J Cardiac Fail. (2019) 25:S267. doi: 10.1016/j.cardfail.2019.07.073

18. Yunis A, Seese L, Stearns B, Genuardi M, Thoma F, Kilic A. Direct oral anticoagulants are effective therapy in treating left ventricular thrombi. J Am Coll Cardiol. (2020) 75:948. doi: 10.1016/S0735-1097(20)3 $1575-8$

19. He J, Ge H, Dong JX, Zhang W, Kong LC, Qiao ZQ, et al. Rationale and design of a prospective multi-center randomized trial of EARLY treatment by rivaroxaban versus warfarin in ST-segment elevation MYOcardial infarction with left ventricular thrombus (EARLY-MYO-LVT trial). Ann Transl Med. (2020) 8:392. doi: 10.21037/atm.2020.02.117

20. Robinson A, Ruth B. Direct oral anticoagulants compared to warfarin for left ventricular thrombi: a single center experience. J Am Coll Cardiol. (2018) 71:981. doi: 10.1016/S0735-1097(18)31522-5

21. Robinson AA, Trankle CR, Eubanks G, Schumann C, Thomspon P, Wallace $\mathrm{R}$, et al. Direct oral anticoagulants versus warfarin for left ventricular thrombi: the RED VELVT study. Circulation. (2019) 140:A13756.

22. Alizadeh M, Antoniou S, Fhadil S, Rathod R, Guttmann O, Knight C, et al. The use of direct oral anti-coagulations (DOACs) compared to vitamin $\mathrm{k}$ antagonist in patients with left ventricular thrombus after acute myocardial infarction. Euro Heart J. (2019) 40:4026. doi: 10.1093/eurheartj/ehz746.1020

23. Daher J, Da Costa A, Hilaire C, Ferreira T, Pierrard R, Guichard JB, et al. Management of left ventricular thrombi with direct oral anticoagulants: 
retrospective comparative study with vitamin $\mathrm{K}$ antagonists. Clin Drug Investig. (2020) 40:343-53. doi: 10.1007/s40261-020-00898-3

24. Robinson AA, Trankle CR, Eubanks G, Schumann C, Thompson P, Wallace RL, et al. Off-label use of direct oral anticoagulants compared with warfarin for left ventricular thrombi. JAMA Cardiol. (2020) 5:68592. doi: 10.1001 /jamacardio.2020.0652

25. Asinger RW, Mikell FL, Elsperger J, Hodges M. Incidence of left-ventricular thrombosis after acute transmural myocardial infarction. Serial evaluation by two-dimensional echocardiography. N Engl J Med. (1981) 305:297302. doi: 10.1056/NEJM198108063050601

26. Robinson AA, Jain A, Gentry M, McNamara RL. Left ventricular thrombi after STEMI in the primary PCI era: a systematic review and meta-analysis. Int $J$ Cardiol. (2016) 221:554-9. doi: 10.1016/j.ijcard.2016.07.069

27. Pöss J, Desch S, Eitel C, de Waha S, Thiele H, Eitel I. Left ventricular thrombus formation after ST-segment-elevation myocardial infarction: insights from a cardiac magnetic resonance multicenter study. Circ Cardiovasc Imaging. (2015) 8:e003417. doi: 10.1161/CIRCIMAGING.115.003417

28. Ibanez B, James S, Agewall S, Antunes MJ, Bucciarelli-Ducci C, Bueno H, et al. 2017 ESC Guidelines for the management of acute myocardial infarction in patients presenting with ST-segment elevation: The Task Force for the management of acute myocardial infarction in patients presenting with STsegment elevation of the European Society of Cardiology (ESC). Eur Heart J. (2018) 39:119-77. doi: 10.1093/eurhearti/ehx393

29. Holbrook AM, Pereira JA, Labiris R, McDonald H, Douketis JD, Crowther M, et al. Systematic overview of warfarin and its drug and food interactions. Arch Intern Med. (2005) 165:1095-106. doi: 10.1001/archinte.165.10.1095

30. Hoffman M, Monroe DM. Impact of non-vitamin $\mathrm{k}$ antagonist oral anticoagulants from a basic science perspective. Arterioscler Thromb Vasc Biol. (2017) 37:1812-18. doi: 10.1161/ATVBAHA.117.306995

31. Eikelboom JW, Connolly SJ, Brueckmann M, Granger CB, Kappetein AP, Mack MJ, et al. Dabigatran versus warfarin in patients with mechanical heart valves. N Eng J Med. (2013) 369:1206-14. doi: 10.1056/NEJMoa1300615
32. Lip GY, Hammerstingl C, Marin F, Cappato R, Meng IL, Kirsch B, et al. Left atrial thrombus resolution in atrial fibrillation or flutter: results of a prospective study with rivaroxaban (X-TRA) and a retrospective observational registry providing baseline data (CLOT-AF). Am Heart J. (2016) 178:126-34. doi: 10.1016/j.ahj.2016.05.007

33. Delewi R, Zijlstra F, Piek JJ. Left ventricular thrombus formation after acute myocardial infarction. Heart. (2012) 98:17439. doi: 10.1136/heartjnl-2012-301962

34. Di Minno A, Spadarella G, Prisco D, Scalera A, Ricciardi E, Di Minno G. Antithrombotic drugs, patient characteristics, and gastrointestinal bleeding: clinical translation and areas of research. Blood Rev. (2015) 29:33543. doi: 10.1016/j.blre.2015.03.004

35. Cannon CP, Bhatt DL, Oldgren J, Lip GYH, Ellis SG, Kimura T, et al. Dual antithrombotic therapy with dabigatran after PCI in atrial fibrillation. N Engl J Med. (2017) 377:1513-24. doi: 10.1056/NEJMoa17 08454

36. Lopes RD, Heizer G, Aronson R, Vora AN, Massaro T, Mehran R, et al. Antithrombotic therapy after acute coronary syndrome or PCI in atrial fibrillation. N Engl J Med. (2019) 380:1509-24. doi: 10.1056/NEJMoa18 17083

Conflict of Interest: The authors declare that the research was conducted in the absence of any commercial or financial relationships that could be construed as a potential conflict of interest.

Copyright $\odot 2021$ Xuan, Chen, Dai, Zhou, Jiang and Zhou. This is an open-access article distributed under the terms of the Creative Commons Attribution License (CC $B Y)$. The use, distribution or reproduction in other forums is permitted, provided the original author(s) and the copyright owner(s) are credited and that the original publication in this journal is cited, in accordance with accepted academic practice. No use, distribution or reproduction is permitted which does not comply with these terms. 